\title{
Reflets
}

Revue d'intervention sociale et communautaire

\section{L'insertion professionnelle des migrants francophones à Ottawa : un processus à double face}

\section{Valérie Fortier, Michelle Tabor et Stéphanie Garneau}

Volume 24, numéro 1, printemps 2018

URI : https://id.erudit.org/iderudit/1051525ar

DOI : https://doi.org/10.7202/1051525ar

Aller au sommaire du numéro

Éditeur(s)

Reflets, Revue d'intervention sociale et communautaire

ISSN

1203-4576 (imprimé)

1712-8498 (numérique)

Découvrir la revue

Citer cet article

Fortier, V., Tabor, M. \& Garneau, S. (2018). L'insertion professionnelle des migrants francophones à Ottawa : un processus à double face. Reflets, 24(1), 164-174. https://doi.org/10.7202/1051525ar d'utilisation que vous pouvez consulter en ligne. 


\title{
L'insertion professionnelle des migrants francophones à Ottawa : un processus à double face
}

\author{
Valérie Fortier \\ Candidate à la maîtrise, École de service social, Université d'Ottawa \\ Michelle Tabor \\ Candidate à la maîtrise, École de service social, Université d'Ottawa \\ Stéphanie Garneau, Ph. D. \\ Professeure agrégée, École de service social, Université d'Ottawa
}

Dans le cadre du cours Dimensions internationales du service social donné à l'Université d'Ottawa, nous avons été amenées à réaliser un entretien ethnographique avec une intervenante œuvrant auprès des personnes migrantes. L'entretien ethnographique consiste en la prise en compte, dans l'analyse, non seulement des propos de la personne enquêtée, mais aussi des interactions de la chercheuse ou du chercheur avec la personne interviewée dès la prise de contact, ainsi que des observations du contexte de l'entretien (Beaud et Weber, 2003). Nous avons ainsi rencontré une agente d'établissement du Centre des services communautaires Vanier (CSCV) de la ville d'Ottawa, ce qui nous a conduites à nous interroger sur la question de l'insertion professionnelle des nouveaux arrivants et arrivantes, particulièrement ceux et celles de langue française. C'est effectivement en accédant au travail que les personnes migrantes peuvent progressivement parvenir à s'incorporer à la société canadienne (Raza, Beaujot et Woldemicael, 2012).

Il se trouve que plusieurs migrantes et migrants francophones sont intéressés par le fait de vivre dans un environnement bilingue comme Ottawa. Selon une analyse des données du recensement de 2006, réalisée par le Conseil de planification sociale d'Ottawa (2010), il y avait 24175 immigrantes et immigrants ayant le français comme langue maternelle ou d'usage à Ottawa, soit 15,1\% de la population francophone ottavienne. Certains migrants et migrantes francophones originaires d'Afrique subsaharienne disent se sentir plus à l'aise à Ottawa, malgré le fait que l'anglais prédomine comme langue de travail, cela en raison des variations entre leur langue et celle parlée du côté du Québec 
(Veronis, 2015). Mugwaneza (2011) énonce pour sa part que la perspective d'apprendre et de pouvoir mettre en pratique l'anglais est attirante pour les personnes migrantes.

Toujours selon les données de 2006, 10,5 \% des personnes migrantes francophones dans la capitale nationale étaient cependant touchées par le chômage comparativement à $5,9 \%$ de la population canadienne générale (Conseil de planification sociale d'Ottawa, 2010). L'intervenante que nous avons rencontrée a nommé trois principaux enjeux pouvant expliquer cet écart entre le taux de chômage des personnes migrantes et celui des personnes nées au Canada : les exigences du marché du travail, la francophonie en contexte linguistique minoritaire et les normes culturelles. En les évoquant tour à tour, nous comprendrons mieux quelques-unes de ses interventions auprès des nouveaux arrivants et arrivantes, de même que le sens qu'elle accorde à son rôle. Auparavant, présentons brièvement le Centre des services communautaires Vanier ainsi que les profils des personnes migrantes qui s'y présentent.

\section{Le Centre des services communautaires Vanier}

Établi au cœur de Vanier, un quartier historiquement habité par des ouvriers francophones, le Centre des services communautaires Vanier (CSCV) intervient auprès de la population francophone d'Ottawa depuis 1980 (Benali, 2013). Ce quartier transitoire situé à proximité du centre-ville offre des logements à prix abordable, ce qui encourage les personnes migrantes à s'y installer à leur arrivée à Ottawa. De plus, plusieurs institutions et organismes communautaires francophones se trouvent au sein de cette petite communauté en plus d'une multitude d'épiceries et de commerces spécialisés dans les produits internationaux. Cela incite toujours plus de personnes migrantes à s'établir dans le quartier ou à le visiter fréquemment, changeant ainsi le visage de la population du quartier. Le contexte de la capitale nationale canadienne est particulier puisqu'on peut y trouver les ambassades ainsi que " les institutions gouvernementales fédérales " (Mugwaneza, 2011, p. 14).

Le Centre oriente sa mission sur les besoins changeants de la communauté dans laquelle il est situé (Centre des services communautaires Vanier, 2017). Il offre une multitude de services sociocommunautaires comme une clinique juridique, des activités pour la famille, du counselling et des services de recherche d'emploi. Le programme d'établissement s'insère dans la mission globale du Centre en "fonctionnant en français " tout en étant un " centre d'expertise accessible et chaleureux " pour les migrantes et migrants habitant la région (ibid., \$1). Les personnes migrantes sont donc attirées au 
Centre par la possibilité de recevoir un service de qualité entièrement en français, et ce, de leur premier contact avec la réception jusqu'à l'aiguillage vers d'autres services internes et externes au Centre, le cas échéant. Lorsque cela s'impose, l'équipe offre également les services d'établissement dans l'une des nombreuses autres langues que les membres de l'équipe maîtrisent. Le service d'établissement recrute facilement de nouveaux participants et participantes à son programme grâce aux références des personnes satisfaites ayant déjà eu recours à son service. En effet, l'intervenante mentionne qu'elle remet toujours deux cartes professionnelles aux personnes qu'elle rencontre. Ainsi, l'information peut être partagée avec d'autres personnes migrantes pouvant bénéficier de l'aide du Centre.

L'intervenante étant elle-même migrante, elle nous indique avoir profité des services du Centre lors de son arrivée au Canada. Elle a participé notamment aux activités du volet emploi du Centre pour s'intégrer au marché de l'emploi canadien. L'intervenante a obtenu une formation en administration au Canada avant de retourner dans son pays d'origine pendant quelques années, puis d'immigrer à Ottawa. Elle est, depuis quelques années, intervenante dans le domaine de l'établissement, cela dans différents organismes communautaires.

\section{Les profils migratoires}

La nuit était déjà tombée lorsque nous nous sommes dirigées vers l'immeuble qui abrite le CSCV. La grande salle d'accueil du Centre était presque déserte; uniquement deux personnes occupaient des ordinateurs disponibles pour l'utilisation du public. La réception a téléphoné à l'agente d'établissement pour l'informer de notre arrivée au temps prévu. Cette dernière nous a accueillies dans l'aire d'attente et dirigées directement vers son bureau clos, où nous nous sommes assises autour d'une table ronde. Grâce au climat de confiance déjà établi par le fait que l'une de nous connaissait l'intervenante, nous avons rapidement pu entrer dans le vif du sujet. Après un bref rappel de l'objectif de la rencontre, nous avons pu lui poser nos questions préparées préalablement, dont une portant sur les profils migratoires qu'elle côtoie dans son cadre professionnel. En réponse à cette question, l'intervenante a précisé qu'elle travaille auprès des personnes migrantes qui ont obtenu la résidence permanente ou qui ont reçu une lettre d'approbation initiale $\mathrm{du}$ ministère de l'Immigration, des Réfugiés et de la Citoyenneté en réponse à leur demande de résidence permanente, ainsi qu’auprès des personnes réfugiées reçues et des personnes protégées selon l'article 95 de la Loi sur l'immigration. Sa collègue, qui provient d'une agence externe, intervient auprès des autres catégories de personnes migrantes qui ne sont 
pas admissibles à son programme. L'agente répartit les personnes avec qui elle travaille en deux catégories ordinaires : celles qui migrent par choix et celles qui n'ont pas choisi de s'établir au Canada :

"Moi, je les divise en deux catégories; ce sont tous des nouveaux arrivants, mais ils n'ont pas le même bagage ni le même background. Tu vois, les nouveaux arrivants qui viennent avec une certaine éducation, un certain bagage professionnel, et y viennent ici pour [...] accéder à une vie meilleure que ce qu'ils avaient [...] Et d'autres, qui sont là malgré eux, parce qu'ils fuient des situations de guerre. Peut-être ils voulaient pas partir, mais, bon, la sécurité passe avant tout. »

Elle mentionne que les personnes qui ont été forcées de migrer vivent généralement plus de défis en ce qui a trait aux barrières linguistiques. De plus, elle explique qu'elles gardent l'espoir de retourner dans leur pays d'origine. En réponse, l'intervenante leur conseille de "vi[vre] le moment présent " et de se "préparer en conséquence ", c'est-àdire pour un retour éventuel. Cela dit, l'agente d'établissement a principalement parlé des enjeux en lien avec le marché du travail ottavien affectant les personnes migrantes qui ont délibérément choisi de migrer dans la région, ce sur quoi nous allons nous pencher maintenant.

\section{Les défis des travailleuses et travailleurs qualifiés francophones à Ottawa}

Depuis de nombreuses années, le gouvernement canadien a mis en place des politiques pour favoriser les flux migratoires entrants. Ceci a mené à la hausse d'une immigration de type économique (Haince, 2014). Or, le contexte actuel du milieu de travail ottavien, marqué par l'écart entre les visées des politiques migratoires et le fonctionnement du marché local de l'emploi, complique l'insertion professionnelle des personnes migrantes.

\section{Le sentiment d'avoir été flouées}

Lors de notre entretien, l'intervenante explique que plusieurs personnes migrantes s'établissent au Canada avec l'espoir de trouver un travail et une qualité de vie supérieure : "Tu vois, les nouveaux arrivants qui viennent avec une certaine éducation, un certain bagage professionnel, et y viennent ici [...] pour accéder à une vie meilleure que ce qu'ils 
avaient. » L'intervenante évoque les frustrations ressenties par les personnes migrantes qui réalisent qu'il y a un écart entre leurs attentes et ce qu'il est possible de réaliser au Canada. L'intervenante souligne que certaines personnes migrantes se sentent flouées parce qu'elles croyaient que le Canada voulait de leur main-d'œuvre qualifiée, alors que l'objectif du gouvernement consiste à pourvoir les postes délaissés par les Canadiennes et Canadiens.

L'un des enjeux rencontrés lors du processus de recherche d'emploi est la nonreconnaissance des diplômes, des titres et des formations antérieurs. Les expériences professionnelles à l'étranger des personnes migrantes ne sont pas toujours reconnues par les employeurs en dépit de leur niveau de qualification et de certification élevé (Statistique Canada, 2003), comme le montrent aussi nombre d'études empiriques réalisées au Québec ou ailleurs au Canada (Li, 2001; Reitz, 2007a, 2007b; Piché, Renaud et Gingras, 2002; Renaud, 2005; Lenoir-Achdjian, et collab., 2007; Garneau, 2008; Vatz Laaroussi, 2008; Chicha, 2012; Blain, Fortin et Alvarez, 2014). Durant l'entretien, l'intervenante ajoute qu'un bon nombre de personnes migrantes arrivent au pays avec l'espoir de trouver un emploi relatif à leurs expériences professionnelles : "Cette personne elle veut que ses acquis, c'est-à-dire pour les travailleurs [...] les résidents économiques, ils veulent que leur bagage soit reconnu. » Devant une pareille situation, l'intervenante leur donne des informations quant aux possibilités qui s'offrent à elles afin d'obtenir des équivalences canadiennes. Elle doit aussi faire beaucoup d'éducation par rapport au système éducatif nord-américain et aux frais de scolarité qui accompagnent une mise à niveau imprévue. Qui plus est, elle doit les sensibiliser à propos des ordres professionnels qui réglementent certains domaines d'emplois particuliers : "Les gens ne comprennent pas comment un ordre est plus fort que l'université. Donc, tu expliques tout cela, okay? Tu as beau être diplômé de l'Université d'Ottawa, si l'ordre ne te donne pas la licence, tu vas pas exercer. " Enfin, plusieurs personnes migrantes se butent à des employeurs qui exigent une première expérience de travail canadienne avant de les embaucher. Afin de déjouer cette situation inextricable, les personnes migrantes effectuent souvent du travail non rémunéré en tant que bénévoles dans l'objectif d'obtenir des références de travail canadiennes. Bref, l'acquisition d'une expérience de travail canadienne devient primordiale pour les personnes migrantes qui cherchent leur premier emploi au Canada, mais s'avère souvent difficile à obtenir (Sweetman et Warman, 2014). Or, l'agente d'établissement nous affirme que le chômage ou l'acquisition d'un emploi plus précaire occasionne une grande période d'instabilité et de stress financier pour les familles. 


\section{Les exigences linguistiques}

La maitrise d'une des deux langues officielles du Canada est « un des principaux facteurs qui contribuent à la réussite de l'intégration professionnelle» (Mugwaneza, 2011, p. 13). Il s'agit également d'un critère auquel les employeurs et le système canadien accordent beaucoup d'importance lors du processus de sélection (Mohamoud, 2007; Savard et Charbonneau, 2016). Au cours de l'entretien, l'intervenante parle de la maîtrise de l'une des deux langues officielles comme étant sa priorité en intervention auprès des migrantes et migrants à Ottawa, et ce, avant même d'entamer les démarches de recherche d'emploi : «Des fois, ils viennent avec des attentes qui ne peuvent pas être comblées pour le moment. Genre, tu peux avoir quelqu'un qui vient, tu dis "Je veux avoir du travail”, mais il ne parle ni français ni anglais. " Pour composer avec ce type de situation, l'intervenante aborde l'enjeu de manière indirecte en faisant allusion aux normes du travail en matière de santé et sécurité :

"Donc [...] tu [ne] lui dis pas: "Non, tu ne vas pas travailler." Non. Mais, tu sais [...] en tout cas ma technique, je leur dis : "Écoutez, ici c'est une question de santé et sécurité. Personne ne voudrait qu'un employé se fasse du mal parce qu'il n'a pas compris une procédure de travail. Donc, peut-être que ça serait intéressant de commencer par les cours de langue et puis faire une transition [...] plus douce vers [...] l'emploi." "

Effectivement, les employeurs « sont réticents à embaucher des migrants » en raison des risques potentiels que pourraient engendrer «les compétences linguistiques insuffisantes ", telles que "d'éventuelles demandes d'assurance-invalidité ou de prestations de la Commission de la sécurité professionnelle et de l'assurance contre les accidents du travail » (Mohamoud, 2007, p. 39). En abordant la question des langues canadiennes officielles de cette façon, l'intervenante veut amener la personne concernée à " [comprendre] le sens des priorités » en lui expliquant les étapes qu'elle doit d'abord franchir, sans pourtant discréditer le besoin de se trouver un emploi qu'elle exprime.

L'insertion socioprofessionnelle des personnes migrantes est donc gravement entravée en raison des critères linguistiques obligatoires pour l'embauche, mais aussi du manque de services de qualité en français pouvant appuyer les personnes migrantes francophones dans leurs démarches (Savard et Charbonneau, 2016). Le CSCV tâche de répondre au mieux à ce besoin de services. L'intervenante reconnaît toutefois la nécessité pour les personnes migrantes d'apprendre l'anglais pour améliorer leur insertion professionnelle, comme cela a d'ailleurs été remarqué au Québec (Lenoir-Achdjian, et collab., 2007; Vatz 
Laaroussi, 2008). L'agente dirige donc les personnes migrantes vers une école de langue seconde du quartier où elle se déplace une fois par semaine pour faire des interventions et la promotion des services du Centre.

\section{“ Ce sont des p'tites choses culturelles, mais d'une très grande sensibilité "}

Veronis (2015, p. 60) mentionne que « les immigrantes et immigrants poursuivent un idéal d'inclusion insaisissable, qu'ils n'arriveront jamais à atteindre » [notre traduction]. En effet, peu importe depuis combien de temps les migrantes et migrants occupent un emploi au Canada, une majorité d'entre eux « sont perçus par les Canadiens comme étant différents d'eux » en raison de l'altérité raciale que leurs caractéristiques visibles, comme la couleur de la peau et l'accent, présentent (Lafontant, 2009, p. 48; Nagra et Maurutto, 2016).

L'entrevue avec l'agente d'établissement nous a montré que la réussite de l'établissement de la personne migrante est généralement évaluée par les pouvoirs publics et par la perception des personnes migrantes ellesmêmes, suivant l'accès à un emploi rémunéré à la hauteur de leurs compétences (Schellenberg et Maheux, 2007; Lafontant, 2009). C'est pourquoi les personnes migrantes qui ont trouvé un logement et qui ont obtenu les documents les autorisant à travailler, puis qui n'ont pas à recourir à un cours de langue ou à une mise à niveau éducative, insistent auprès de l'agente d'établissement pour trouver un emploi afin de commencer à travailler dès que possible. Par contre, selon Madut (2016, p. 94), « la sélection des employées et employés potentiels n’est pas limitée aux compétences et aux habiletés, mais s'étend aussi aux connaissances des normes sociales et de la culture du travail au Canada de la candidate ou du candidat " [notre traduction]. L'agente doit alors jouer un rôle de sensibilisation aux normes canadiennes auprès des personnes migrantes pour qu'elles puissent obtenir un emploi et le conserver.

Lagente mentionne qu'elle prend davantage de temps avec les individus qui sont nouvellement arrivés au pays afin de les initier aux normes à respecter dans la vie quotidienne, mais aussi dans les milieux de travail au Canada. En abordant celles-ci, l'intervenante demeure sensible au vécu des personnes migrantes en tenant compte de la manière dont les choses étaient faites dans leur pays d'origine ou dans les milieux où elles ont vécu lors de leur parcours migratoire. Elle dit qu'elle aborde avec elles :

" [t] oute la dynamique qui encadre comment les choses se font dans le pays d'origine de la personne, et comment ça se fait ici [...] Vivre de manière 
harmonieuse, qu'est-ce que ça prend? C’est durant leur passage ici [que] je profite de ça pour les informer. »

Pour certaines personnes qui ont vécu pendant de longues périodes dans des camps de réfugiés ou d'autres endroits où il y avait peu de structures organisationnelles, elle explique comment les structures et les normes au Canada peuvent être un réel défi. Par exemple, elle nous a parlé de la façon dont elle intervient auprès des individus qui parlent fort dans le corridor en quittant son bureau :

"Les gens qui sont habitués à parler très fort, tu leur dis "okay on va se voir demain” [et qui répondent en criant] : D’ACCORD. Tu leur dis : "Il y a des gens qui travaillent, tu peux les déranger." "

Lors de cette intervention, l'agente d'établissement souhaite sensibiliser les personnes migrantes à une norme sociale en milieu de travail qui est liée au ton de voix et au bruit, et à laquelle elles n'ont probablement pas porté attention. Si ces personnes n'avaient pas été informées, peut-être que ce même comportement aurait pu leur occasionner plus tard certains problèmes relationnels ou les empêcher tout simplement d'obtenir un emploi. Malheureusement, dans d'autres situations, l'apprentissage de certaines normes se fait par une expérience négative :

"Au Canada, c'est très mal vu de parler du poids de quelqu'un. Chez nous [pays d'origine de l'intervenante], si quelqu'un prend du poids [...], c'est bien. Ça veut dire qu'il se sent bien [...] Le monsieur, il a dit à sa boss : "Oh, c'est bien, tu as grossi." "

La compréhension erronée de certaines normes, formelles comme informelles, peut engendrer des faux pas pouvant mener à la perte d'un emploi, comme cela a été le cas pour l'individu dans l'exemple précédent.

\section{Conclusion : l'intégration, une réalité à deux faces}

En somme, l'intervenante a énuméré une panoplie d'obstacles structurels se présentant à différents moments dans le processus d'établissement des personnes migrantes. Comme nous l'avons présenté, le marché du travail, la langue et la culture agissent comme déterminants de l'insertion professionnelle des personnes migrantes, pourtant l'un des besoins les plus pressants lors de leur installation dans la capitale nationale afin d'obtenir 
une stabilité financière. Si certaines parviennent à détenir des emplois valorisants, le chemin est cependant pour la plupart ardu à parcourir.

Il faut donc reconnaître le rôle joué par l'État et la société canadienne par rapport à l'intégration économique des personnes migrantes dans un emploi à la hauteur de leurs compétences. L'agente d'établissement insiste sur l'importance d'être sensibilisés à la réalité des personnes migrantes et de miser sur la coexistence et la collaboration :

"Moi, je pense que c'est une préparation à un double niveau : préparer la communauté d'accueil et préparer les personnes qui arrivent [...] Ça aurait été bien de faire beaucoup de, comment dire, beaucoup d'ateliers sur le vivre ensemble, c'est à dire comprendre l'autre."

On peut se demander si, à l'heure actuelle, on ne tend pas à considérer les personnes migrantes comme les seules responsables de leur intégration. Or, l'engagement de la communauté d'accueil est tout aussi nécessaire. La transformation du discours et des pratiques est primordiale afin qu'ils misent sur la valeur ajoutée des personnes migrantes au sein de l'économie canadienne.

\section{Bibliographie}

BEAUD, Stéphane, et Florence WEBER (2003). Guide de l'enquête de terrain, Paris, La Découverte, p. 183-184, p. 188-202 et p. 203-230.

BENALI, Kenza (2013). «La densification urbaine dans le quartier Vanier : germe d'un renouveau urbain ou menace pour le dernier îlot francophone de la capitale canadienne? ", Cahiers de géographie du Québec, Vol. 57, N 160, p. 41-68.

BLAIN, Marie-Jeanne, Sylvie FORTIN et Fernando ALVAREZ (2014). «Être médecin et immigrant au Québec, une identité professionnelle malmenée ", Revue européenne des migrations internationales, Vol. 30, No 3-4, p. 139-162.

CENTRE DES SERVICES COMMUNAUTAIRES VANIER (2017). Mission, valeurs et visions, réf. du 14 avril 2017, http://www.cscvanier.com/fr/centre/a-propos/mission

CHICHA, Marie-Thérèse (2012). « Discrimination systémique et intersectionnalité : la déqualification des immigrantes à Montréal ", Revue de la femme et le droit/Canadian Journal of Women \& the Law, Vol. 24, № 1, p. 82-113.

CONSEIL DE PLANIFICATION SOCIALE D'OTTAWA (2010). Profil des immigrants francophones à Ottawa, Ottawa, $27 \mathrm{p}$. 
GARNEAU, Stéphanie (2008). «L'émigration marocaine au Canada : contextes de départ et diversité des parcours migratoires ", Diversité urbaine, Vol. 8, No 2, p. 163-190.

HAINCE, Marie-Claude (2014). "La domestication de l'Autre ou la fabrication de l'“immigrant parfait" ", Diversité urbaine, Vol. 14, № 1, p. 95-116.

LAFONTANT, Jean (2009). "Travail, identité et territorialité : l'immigration subsaharienne dans les communautés francophones en situation minoritaire au Canada ", dans M. Labelle et F. W. Remiggi (dirs.), Inégalités, racisme et discriminations : regards critiques et considérations empiriques, Université du Québec à Montréal, Les Cahiers de la CRIEC, p. 39-58.

LENOIR-ACHDJIAN, Annick, et collab. (2007). "The Professional Insertion of Immigrants Born in the Maghreb : Challenges and Impediments for Intervention ", International Migration \& Integration, Vol. 8, p. 391-409.

LI, Peter S. (2001). "The Market Worth of Immigrants' Educational Credentials », Canadian Public Policy, Vol. 27, p. 23-38.

MADUT, Kon (2016). « How do racialized migrants cope with challenges of labor market integrations and resettlement? ", Amsterdam Social Science, Vol. 7, № 1, p. 93-110.

MOHAMOUD, Hindia (2007). "L'immigration et la composition socioculturelle et démographique d'Ottawa ", Institut Métropolis, № 4, p. 33-43.

MUGWANEZA, Théodésie (2011). Femmes immigrantes et insertion en emploi : parcours et analyse des trajectoires des Rwandaises dans la région d'Ottawa-Gatineau, [mémoire de maîtrise], École de développement international et mondialisation (ÉDIM), Faculté des sciences sociales, Université d'Ottawa, $165 \mathrm{p}$.

NAGRA, Baljt, et Paula MAURUTTO (2016). «Crossing Borders and Managing Racialized Identities: Experiences Of Security And Surveillance Among Young Canadian Muslims », Canadian Journal of Sociology, Vol. 41, № 2, p. 165-194.

PICHÉ, Victor, Jean RENAUD et Lucie GINGRAS (2002). «L'insertion économique des nouveaux immigrants dans le marché du travail à Montréal : une approche longitudinale ", Population, Vol. 57, No 1, p. 63-89.

RAZA, Muhammad, Roderic BEAUJOT et Gebremariam WOLDEMICAEL (2012). "Social Capital and Economic Integration of Visible Minority Immigrants in Canada ", Journal of International Migration and Integration, Vol. 14, № 2, p. 263-285.

REITZ, Jeffrey G. (2007a). "Immigrant Employment Success in Canada, Part I: Individual and Contextual Causes », Journal of International Migration \& Integration, Vol. 8, № 1, p. 11-36.

REITZ, Jeffrey G. (2007b). «Immigrant Employment Success in Canada, Part II: Understanding the Decline ", Journal of International Migration \& Integration, Vol. 8, № 1, p. 37-62. 
RENAUD, Jean (2005). "Limites de l'accès à l'emploi et intégration des immigrants au Québec : quelques exemples à partir d'enquêtes ", Santé, société et solidarité, No 1, p. 35-46.

SAVARD, Sébastien, et Dominique CHARBONNEAU (2016). "Réalité, enjeux et défis de la pratique du développement communautaire auprès des communautés francophones en situation minoritaire ", Reflets : revue d'intervention sociale et communautaire, Vol. 22, No 2, p. 69-94.

SCHELLENBERG, Grant, et Hélène MAHEUX (2007). « Perspectives des immigrants sur leurs quatre premières années au Canada : Faits saillants des trois vagues de l'Enquête longitudinale auprès des immigrants du Canada ", Tendances sociales canadiennes, Ottawa, Statistique Canada, $117 \mathrm{p}$.

STATISTIQUE CANADA (2003). Enquête longitudinale auprès des immigrants du Canada : Le processus, les progrès et les perspectives, réf. du 12 avril 2017,

http://www.statcan.gc.ca/access_acces/alternative_alternatif.action?l=fra\&loc=/pub/89-611-x/89611-x2003001-fra.pdf

SWEETMAN, Arthur, et Casey WARMAN (2014). "Former Temporary Foreign Workers and International Students as Sources of Permanent Immigration ", Canadian Public Policy / Analyse de Politiques, Vol. 40, № 4, p. 391-407.

VATZ LAAROUSSI, Michèle (2008). " Du Maghreb au Québec : accommodements et stratégies », Travail, genre et sociétés, Vol. 2, No 20, p. 47-65.

VERONIS, Luisa (2015). «Immigrants' Narratives of Inclusion and Belonging in the Transborder City of Ottawa-Gatineau, Canada's National Capital Region ", Canadian Ethnic Studies, Vol. 47, $\mathrm{N}^{\circ} 2$, p. $45-65$. 